\title{
DNA Methylation Pattern of CALCA and CALCB in Extremely Premature Infants with Monochorionic Triplets after Single- Embryo Transfer
}

\author{
Feng Gao, ${ }^{1}$ Yujia Guo, ${ }^{2}$ Xingting Chen, ${ }^{2}$ Qiuyang Gu, ${ }^{3}$ Shirong Huang $\mathbb{D}^{4}$ \\ Qingquan Chen $\left({ }^{4},{ }^{4}\right.$ Xiaoming Xu, ${ }^{5}$ Kai Zeng $\odot{ }^{6}{ }^{6}$ Huilin Zhou, ${ }^{2}$ Yilu Zou, ${ }^{2}$ and Qicai Liu $\varpi^{2}$ \\ ${ }^{1}$ Department of Pathology, 1st Affiliated Hospital, Fujian Medical University, Fuzhou 350004, China \\ ${ }^{2}$ Center for Reproductive Medicine, 1st Affiliated Hospital, Fujian Medical University, Fuzhou 350004, China \\ ${ }^{3}$ Department of Medical Ultrasonography, 1st Affiliated Hospital, Fujian Medical University, Fuzhou 350005, China \\ ${ }^{4}$ Department of Laboratory Medicine, Fujian Medical University, 350004 Fuzhou, China \\ ${ }^{5}$ Beijing Perfect Family Hospital, Beijing 100032, China \\ ${ }^{6}$ Department of Anesthesiology, 1st Affiliated Hospital, Fujian Medical University, Fuzhou 350005, China
}

Correspondence should be addressed to Qicai Liu; lqc673673673@163.com

Feng Gao, Yujia Guo, Xingting Chen, and Qiuyang Gu contributed equally to this work.

Received 29 May 2021; Revised 15 August 2021; Accepted 5 September 2021; Published 5 October 2021

Academic Editor: Mariaurea Matias Sarandy

Copyright ( $) 2021$ Feng Gao et al. This is an open access article distributed under the Creative Commons Attribution License, which permits unrestricted use, distribution, and reproduction in any medium, provided the original work is properly cited.

Compared with full-term peers, premature infants are more likely to suffer from neonatal diseases and death. Variations in DNA methylation may affect these pathological processes. Calcitonin gene-related peptide (CGRP) plays a complex and diversified role in reproduction and chronic inflammation, and participates in the functional maintenance of vascular adaptation and trophoblast cells during pregnancy. Here, premature live births with single-chorionic triple embryos after single-embryo transfer were used as research objects, while full-term infants with double embryos and double-chorionic twins were used as controls. DNA was extracted from umbilical cord tissues for pyrosequencing to detect the methylation level of CpG island in CGRP promoter region. The average values of CGRP methylation in the umbilical cord tissues of very premature fetuses were higher than that of normal controls obtained from the databases. Immunofluorescence results showed that the expression of $\alpha$ CGRP was decreased in the blood vessel wall of the umbilical cord of monozygotic triplets, especially in death cases, while the $\beta$ CGRP had a compensatory expression. In conclusion, our findings suggest that hypermethylation of CGRP might be considered as an important cause of serious neonatal morbidities.

\section{Introduction}

Impaired blood circulation in the umbilical cord or placenta might lead to intrauterine growth restriction and premature delivery and is considered as an important complication during pregnancy [1]. Calcitonin gene-related peptide (CGRP) is regarded as the most effective vasodilator, and it plays a complex and diversified role in chronic low-grade inflammation [2]. It is closely related to functional maintenance of vascular adaptation and trophoblast cells during pregnancy [3]. It comprises two subtypes CALCA ( $\alpha$ CGRP) and CALCB ( $\beta$ CGRP), and these coordinate with molecular signaling network of decidualization, placenta formation, and fetal growth to promote successful pregnancy outcomes $[3,4]$. But the role of it in finely synchronized molecular and cellular events during preterm labor still remains to be unknown.

Our previous studies have revealed that abnormal secretion of $\beta$ CGRP is caused by CALCB mutation, which lose its inhibitory effect on inflammatory cells, resulting in obliterative vasculitis and perineuritis [5]. At the same time, interleukin- (IL-) 6, IL-10, and tumor necrosis factor- $\alpha$ (TNF- 
$\alpha$ ) were highly expressed in CGRP-KO rats, which might be involved in the occurrence of fetal inflammation [6]. CGRP can inhibit the synthesis and/or release of inflammatory factors such as TNF- $\alpha$, IL- 1 , and IL- 12 by regulating cytokine expression of macrophages through cAMP/PKA signaling pathway and inhibiting the differentiation of Th1 lymphocytes. CGRP can also regulate the balance between Th1/Th2 cells, promoting the release of IL-10 and IL- 4 from macrophages and Th2 lymphocytes and inhibiting the antigen presentation of Th1 cells, thereby inhibiting Th1 cell-mediated cellular immunity $[7,8]$. Therefore, we speculated that CGRP might participate in immune regulation and inhibition of inflammation during pregnancy, thereby affecting fetal development and progression of labor.

In addition to the influence of neurological factors, CGRP expression also plays an important regulatory role in its own gene structure and methylation modification. There is a large $\mathrm{CpG}$ island in the $5^{\prime}$ flanking promoter region of the CALCA and contains a microbial infectionspecific response elements that regulate the transcription of procalcitonin (PCT) during bacterial infection [9]. The structure of CALCB is similar to that of CALCA, and it consists of two different CpG-rich regions, wherein one is located around exon 1, and the other is located about $1.5 \mathrm{~kb}$ upstream [10]. Single-egg multiple births might occur due to different methylation patterns during embryonic development, and this might be a reason for the differences in fetal development [11]. The monochorionic triplet (MCTA) genetic background is a common, reliable, and excellent sample for studying the role of CGRP in pregnancy maintenance. Hence, in this study, the methylation levels and expression location of CALCA and CALCB in the umbilical cord tissues of monochorionic three amniotic sacs were detected, and the relationship between them and the very preterm birth were evaluated.

\section{Materials and Methods}

2.1. Research Subject. A case of monochorionic triplets and the very premature live births of three boys after single blastocyst transplantation were collected as research subjects from the Reproductive Medicine Center of the First Affiliated Hospital of Fujian Medical University in April 2020. In addition, those undergoing double blastocyst transplantation in the same period were selected. Chorionic twins and two full-term live births were used as controls. General information including age, basic hormone levels, fetal development process, and family history were collected. After obtaining their consent, the remaining umbilical cord and placental tissue samples from in vitro pathological diagnosis were collected for methylation and immunohistochemical testing.

2.2. Database Retrieval and Prediction. To study the relationship between gene methylation in umbilical cord or cord blood and preterm birth, two matching databases were found. Genome wide DNA methylation profiling of 152 umbilical cord blood samples from CCCEH birth cohort. The Illumina Infinium 450k Human DNA methylation
Beadchip was used to obtain DNA methylation profiles across approximately 450,000 CpGs in cord blood samples. https://www.ncbi.nlm.nih.gov/geo/query/acc.cgi?acc= GSE69176.

An Epigenetic-Senescence-Signature, based on six CpGs, was either analyzed by pyrosequencing or by barcoded bisulfite amplicon sequencing. https://www.ncbi.nlm.nih.gov/ geo/query/acc.cgi?acc $=$ GSE82234.

2.3. Pyrosequencing. DNA was extracted from umbilical cord tissues (about $5 \mathrm{~cm}$ from placenta attachment) of three infants with single chorionic triplets and preterm live birth after single blastocyst transplantation and double blastocyst transplantation double chorionic twins and two full-term live births using the Tiangen DNA Extraction Kit (TIANGEN, Beijing, China) according to manufacturer's instructions. DNA was sent directly to Gene Tech (Shanghai) Co, Ltd. (Gene Tech, Shanghai, China) for pyrosequencing to detect the methylation level of CpG island in CGRP promoter region. The sequencing primers, conditions, and sequencing sequences used are shown in Figure 1.

2.4. Immunofluorescence. The immunofluorescence measurements were performed as previously described [12]. The procedure is briefly described as follows: umbilical cord tissue and placental tissue were fixed in $4 \%$ formalin overnight, embedded in paraffin, sectioned at $4 \mathrm{~mm}$. Immunofluorescence confocal microscopy was also undertaken to determine the correlation of CALCA $(\alpha C G R P)$ and CALCB $(\beta C G R P)$. CALCA was detected with rabbit antihuman polyclonal antibody (1:50 dilution; A11804, Abclonal, $\mathrm{CN}$ ), and CALCB was detected with rabbit antihuman polyclonal antibody ( $1: 200$ dilution; A5523, Abclonal, CN). The secondary antibody was rhodamine- (TRI-TC-) conjugated goat anti-rabbit IgG or FITC-labeled goat anti-rabbit IgG. Nuclei were stained with DAPI solution.

\section{Results}

3.1. Clinical Data of Identical Triple Pregnancies. A 29-yearold female patient was treated with in vitro fertilization (IVF) and transplanted a fresh cycle of one blastocyst $(4 \mathrm{AB})$ on day 5 , which is a monochorionic triple amniotic pregnancy shown in pregnancy ultrasound. This blastocyst was premature rupture of membranes at $25 \mathrm{~W}+1$ week, and three boys were born prematurely. The newborns had an Apgar score of 4 and a weight of $800 \mathrm{~g}$. One of the newborns was short in height and malnourished and died after rescue (Table 1). General clinical data show that the patient has lived with the current husband for 6 years, married for 5 years, and has an average sex life of 3-4/month. The husband has ejaculated and has not been pregnant yet. The patient was diagnosed with "polycystic ovary syndrome" in 2015. In 2016, the patient underwent a series of operations, such as laparoscopic ovarian perforation, hysteroscopy, and bilateral salpingectomy. Patient's infusion during surgery is normal. The pathological results after surgery showed endometrial polyps. Basic endocrine examination: $\mathrm{FSH}$ $1.11 \mathrm{IU} / \mathrm{L}, \mathrm{LH} 6.4 \mathrm{IU} / \mathrm{L}, \mathrm{E} 235.2 \mathrm{miu} / \mathrm{L}, \mathrm{T} 57.6 \mathrm{nmol} / \mathrm{L}$, and 
CALCA

\begin{tabular}{|l|l|}
\hline Primer Set 1 & Score: 62 \\
\hline General Warnings & \\
\hline
\end{tabular}

\begin{tabular}{|l|l|l|}
\hline $\mathbf{}$ & F1 & TTATAGTTTYGGGATGTAGTTGTTG \\
\hline \hline & R1 & TTTCAACCTCTCCACCATCT \\
\hline$\rightarrow$ & S1 & TGTTGAGTTTATYGTATAGGTA \\
\hline
\end{tabular}

\begin{tabular}{|l|l|l|l|l|}
\hline \multirow{2}{*}{ Length, bp } & PCR Product & Forward PCR Primer, F1 & Reverse PCR Primer, R1 & Sequencing Primer, S1 \\
\cline { 2 - 5 } Position, $5^{\prime} 3^{\prime}$ & 109 & 25 & 20 & 22 \\
\hline Tm, ${ }^{\circ} \mathrm{C}$ & & $538-562$ & $646-627$ & $558-579$ \\
\hline \%GC & & 65.4 & 45.1 & 45.9 \\
\hline Sequence to Analyze & GYGTTYGGAT YGGTTGTAGT AGATYGYGYG TTGYGYGTTT TATYGGGAGA TGGTGGAGA \\
\hline
\end{tabular}

\section{CALCB}

\begin{tabular}{|l|l|}
\hline Primer Set 0 & Score: 79 \\
\hline GeneralWarnings & \\
\hline
\end{tabular}

\begin{tabular}{|l|l|l|}
\hline $\mathbf{2}$ & F1 & GGTCAGTTTTGGTTATTTTGGAT \\
\hline \hline $\mathbf{2}$ & R1 & CCTCACCTAACCACTAAAATTTCA \\
\hline$\rightarrow$ & S1 & TTTTTAGTTATTTTTGTAATAAG \\
\hline
\end{tabular}

\begin{tabular}{|l|l|l|l|l|}
\hline & PCR Product & Forward PCR Primer, F1 & Reverse PCR Primer, R1 & Sequencing Primer, S1 \\
\hline Length, bp & 175 & 23 & 24 & 23 \\
\hline Position, 5' $3^{\prime}$ & & $184-206$ & $358-335$ & $236-258$ \\
\hline Tm, ${ }^{\circ} \mathrm{C}$ & & 67.1 & 67.4 & 47.6 \\
\hline \%GC & 34.8 & 37.5 & 13.0 \\
\hline Sequence to Analyze & $\begin{array}{l}\text { AGYGGGGTTT TYGYGGGGAA GGYGTTTATA GTAGGTGTGG TGTTTATTTY GGGTYGATYG } \\
\text { GTYGTTYGYG TTGTTTTGAA ATTTTAGT }\end{array}$ & \\
\hline
\end{tabular}

FIGURE 1: Primers, conditions, and sequencing sequences of CALCA and CALCB methylation.

AMH $5.95 \mathrm{ng} / \mathrm{mL}$. Birth history: 0-0-0-0. Not pregnant for 6 years without contraception. The semen of the man is normal, the chromosomes of both men and women are normal, and the close relatives of both men and women do not have multiple births. All human studies were approved by the Ethics Committee of the First Affiliated Hospital of Fujian Medical University (MTCA, ECFAH of FMU [2019] 001) and (MTCA, ECFAH of FMU [2015] 084-1).

2019.8.23 Ultrasound showed 5 antral follicles on the left ovary, $27 * 22 \mathrm{~mm}$ follicles on the right ovary, and 3-4 antral follicles, Em: $13.0 \mathrm{~cm}$, C Type. GnRH-a ultralong regimen was used, and leuprolide was adjusted down $3.75 \mathrm{mg} / \mathrm{d}$ after communication with the patient. 2019.9.10 Leuprolide was used to downregulate for 27 days, blood hormone: E2: 33.19 pmol/l, FSH: 2.42 IU/L, LH: $0.51 \mathrm{IU} / \mathrm{L}, P$ : $0.64 \mathrm{nmol} / \mathrm{L}$. Vaginal B-ultrasound shows that 8-9 antral follicles can be seen on the left ovary, 4 antral follicles can be seen on the right ovary, Em: $2.0 \mathrm{~mm}$, C Type. After communicating with the patient, she was given LH 150 IU/day and HMG225 IU/d growth hormone 4 IU/d started. 2019-9-30 Gn11day, vaginal B-ultrasound: there are 22 follicles in both ovaries, of which 6 are $>1.8 \mathrm{~cm}$ follicles. Blood hormone: E2: $7678 \mathrm{pmol} / \mathrm{L}, P$ $1.11 \mathrm{nmol} / \mathrm{L}$, LH: $1.25 \mathrm{IU} / \mathrm{L}$. Stop Gn, Azer 200ug trigger, OPU operation for 38 hours. 12 eggs were examined, 10 of which were mature. IVF short-term fertilization, seven appeared 2PN, and D3 high-quality 3 embryos. A fresh cycle embryo is transferred and administered: dydrogesterone
$20 \mathrm{mg}$ bid po*10 d; progesterone suppository 2 tabletsbid $\mathrm{pv} * 10 \mathrm{~d}$.

Frozen embryos: D5 (4AB, 4BB), D6 (4CC+).

Fresh cycle embryo transfer: single blastocyst transplantation (2PN-4CII-8CII-4BA) (Figure 2(a)), the transplant process went smoothly. Day 11, blood HCG $194.7 \mathrm{mIu} / \mathrm{mL}$, day 14, blood HCG $595.1 \mathrm{mIu} / \mathrm{mL}, 6 \mathrm{~W}+4$ ultrasound shows gestational sac size: $3.5 * 1.4 * 2.3(\mathrm{~cm})$. Gross pathology showed single placenta, three amniotic sacs, and three umbilical cords (Figures 2(b) and 2(c)). Histopathology showed acute chorioamnionitis and chorionic plate vasculitis (Figure 2(d)). No triplet transfusion syndrome was found.

3.2. Ultrasound Monitoring Results. Early ultrasound (12 W) of three independent fetuses ( $a, b$, and $c)$, a single placenta, and three independent amniotic sacs were shown in Figure 3(a). Color Doppler ultrasound NT examination revealed three fetuses and one placental echo in the uterine cavity, and the three fetuses were arranged in the upper right (a), upper left (b), and lower right (c) quadrants. Echoes of the diaphragm were seen among the three fetuses, and the three diaphragms and the placenta showed a $\mathrm{T}$ sign. The placenta was located in the posterior wall, covering the cervix by its lower edge. Maturity level was 0 (Figure 3(b)). Ultrasound examination in second trimester (20 weeks) revealed placental maturity level of I and thickness of about $3.23 \mathrm{~cm}$ (Figure 3(c)). 
TABLE 1: General condition of newborns with three births with monochorionic three amniotic sac.

\begin{tabular}{lccccccc}
\hline Fetus & Survival & Apgar score & Weight $(\mathrm{g})$ & Height $(\mathrm{cm})$ & Health status & Birth defects & Other \\
\hline A & Yes & 4 & 806 & 25 & General & None & Healthy discharge \\
B & Yes & 4 & 833 & 25 & General & None & Healthy discharge \\
C & Yes & 4 & 800 & 22 & Unhealthy & None & Died after rescue \\
\hline
\end{tabular}
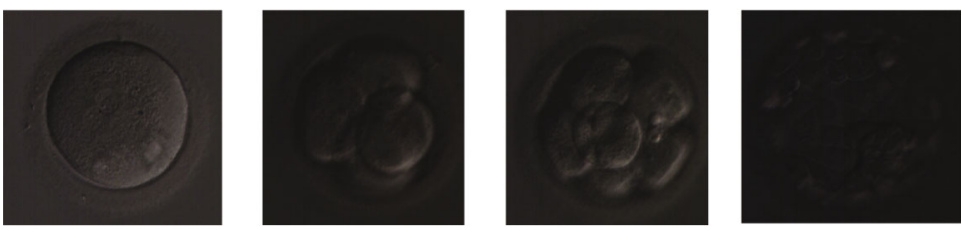

(a)
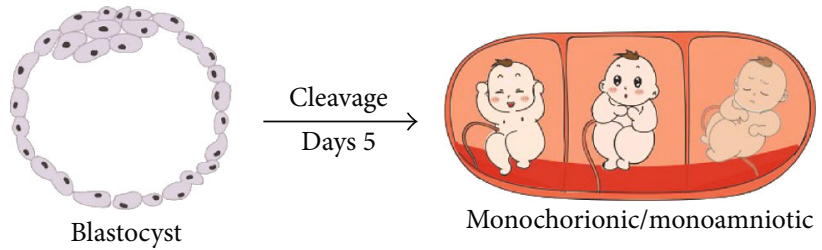

Monochorionic/monoamniotic

(b)

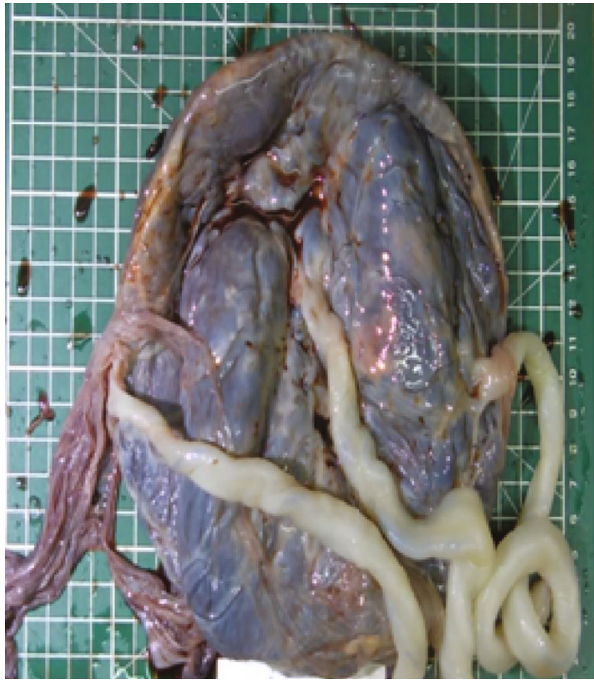

(c)

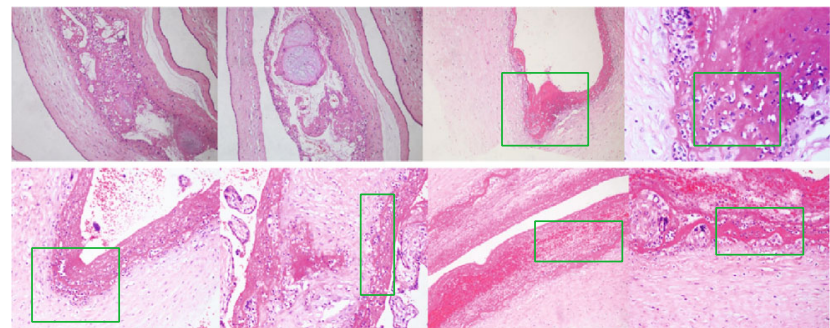

(d)

Figure 2: Clinical data of identical triple pregnancies. (a) Embryo development (2PN-4CII-8CII-4BA), scale 0.0002 mm; (b) identical triplet pattern diagram; (c) identical triple placenta umbilical cord and blood vessels on the umbilical cord (Left: general sample image of placenta and umbilical cord; upper right: pathological image of umbilical cord blood vessel of fetus A; middle right: pathological image of umbilical cord blood vessel of fetus B; lower right: pathological image of umbilical cord blood vessel of fetus); (d) chorioamnionitis and chorionic plate vasculitis, scale bar $0.003 \mathrm{~mm}$; green box marketed and highlight show inflammatory cell infiltration area.

3.3. Database Search Results. The umbilical vein (6 samples) and cord blood (152 samples) were collected from the samples. The average methylation rate of CALCA was $10.08 \%$ and $9.50 \%$, and that of CALCB were $5.31 \%$ and $7.71 \%$, respectively.

3.4. Methylation Sequencing Results. The average values of $\mathrm{CpG}$ island methylation in CALCA promoter region in fetal $\mathrm{A}, \mathrm{B}$, and C umbilical cord tissues were $12.125 \%, 15.5 \%$, and $18.625 \%$, respectively, and these were higher than those in normal controls. The average methylation rate of CALCA promoter region in $\mathrm{D}$ and $\mathrm{E}$ umbilical cord tissues of fullterm control fetuses was only $6.125 \%$ and $4.375 \%$. Based on this, it was clear that the percentage of CALCA methylation in the umbilical cord tissues of premature fetuses was higher than that of full-term births, and the percentage of CALCA methylation in the $\mathrm{C}$ umbilical cord tissues of fetuses that died after rescue remained the highest.

Similarly, the average values of CALCB methylation in umbilical cord tissues of premature fetuses $\mathrm{A}, \mathrm{B}$, and $\mathrm{C}$ were $15.889 \%, 11.222 \%$, and $16.667 \%$, respectively, and were higher than those of normal controls. However, the average 

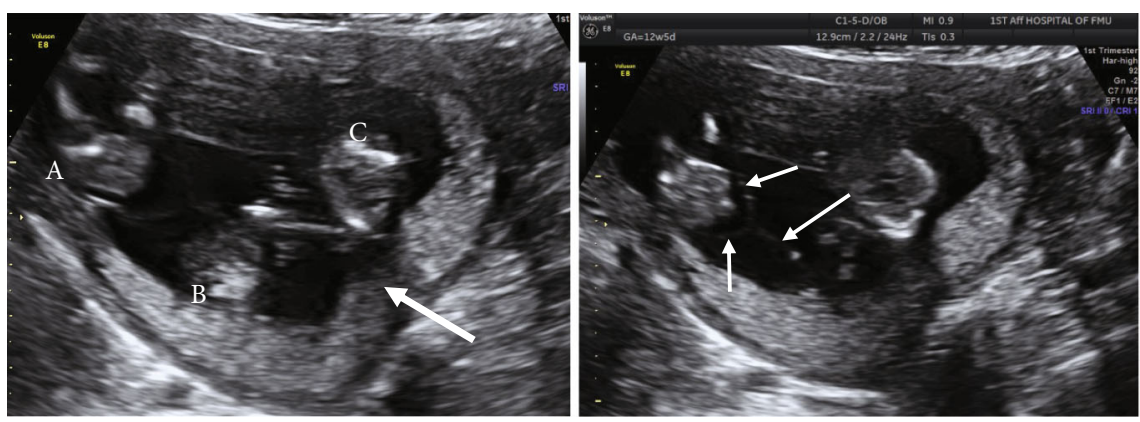

(a)
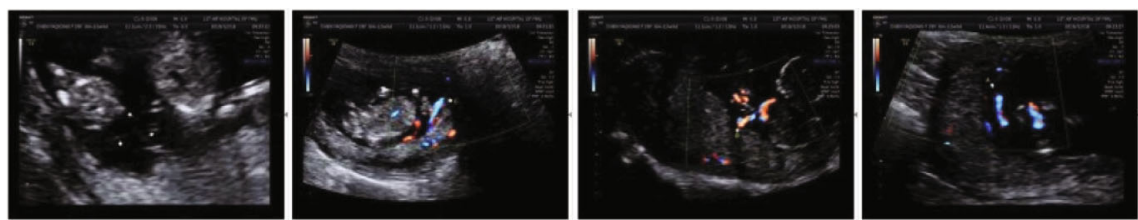

(b)
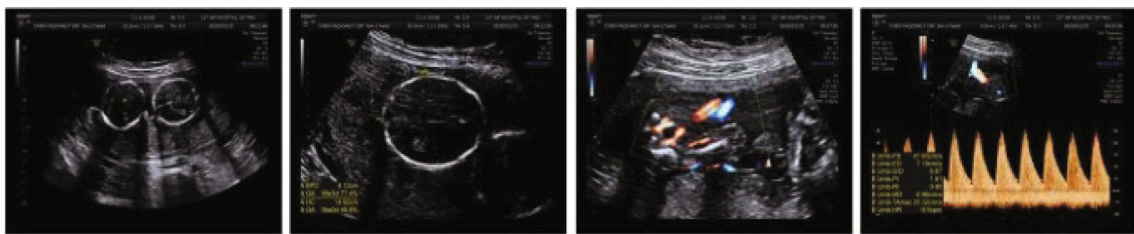

(c)

Figure 3: B-ultrasound monitoring results of identical triple pregnancies. (a) Left: three independent fetuses a, b, and c can be seen, and the arrow is a single placenta; right: three independent amniotic sacs are seen, and the arrow is the amniotic echo; (b) early color Doppler ultrasound NT examination; (c) ultrasonography in the second trimester (20 weeks).

(c)

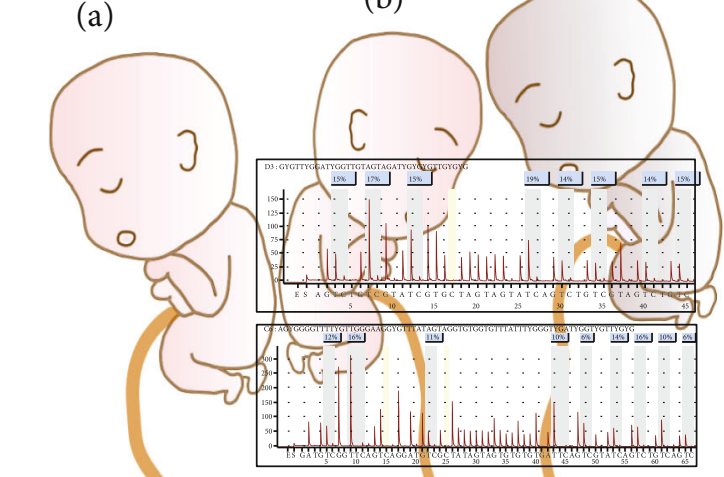

(a)

(b)

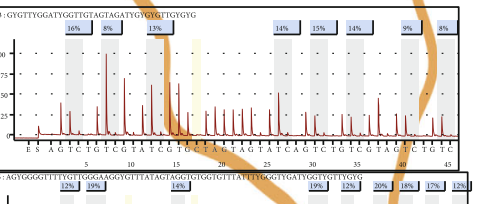

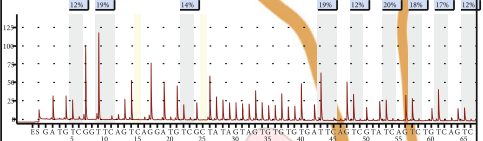

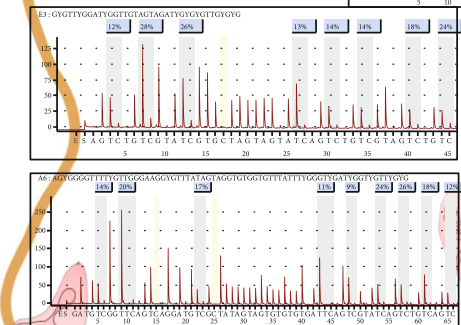

(d)

(e)
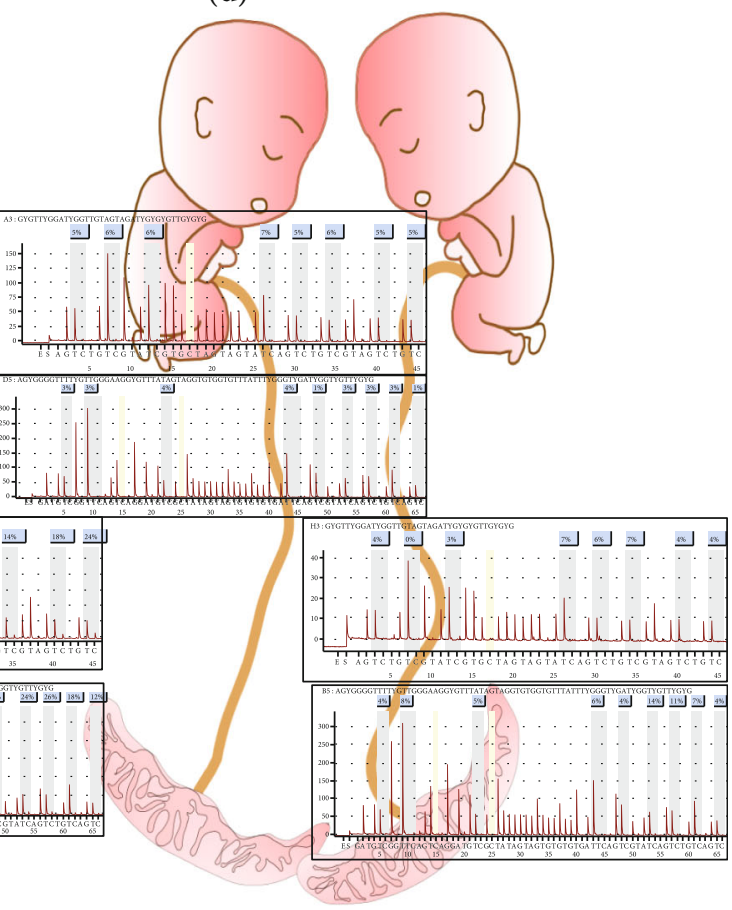

Figure 4: CALCA and CALCB methylation results of umbilical cord tissues from identical triple pregnancy and control. Among them, the identical triplets were triplet (a), triplet (b), and triplet (c) and triplet (d) and triplet (e) fraternal twins. 

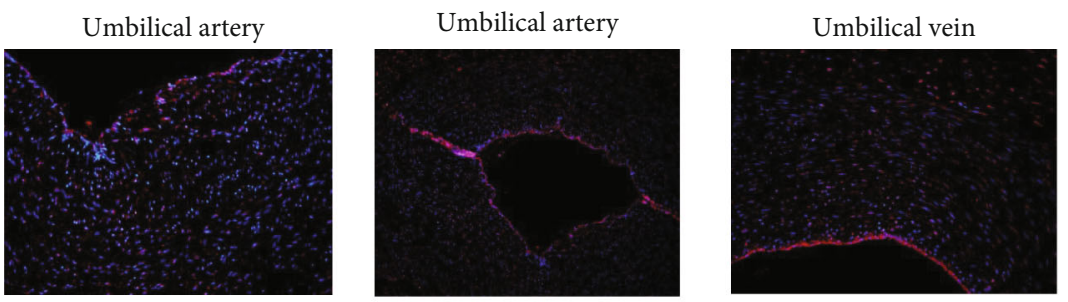

(a)
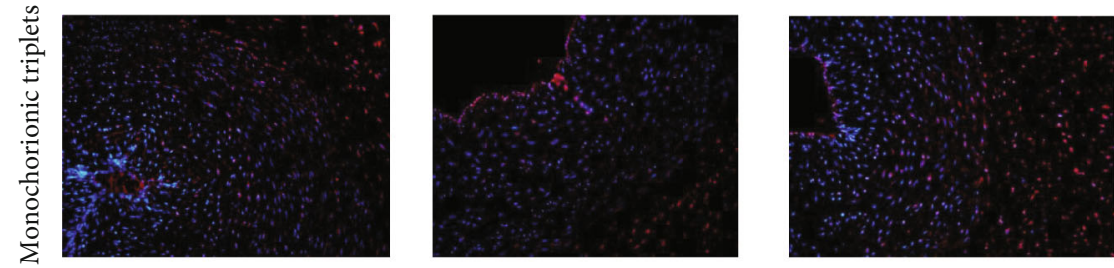

(b)
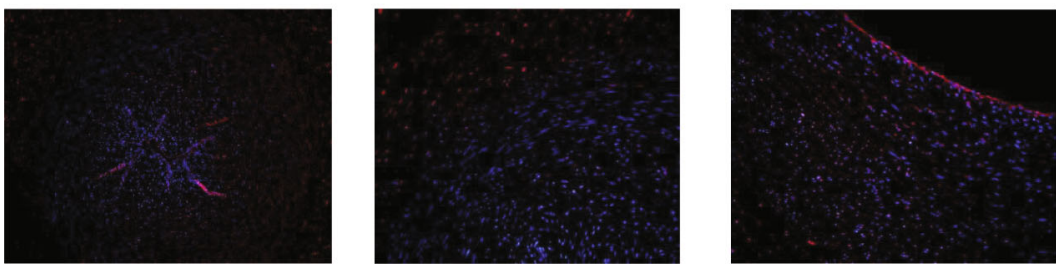

(c)
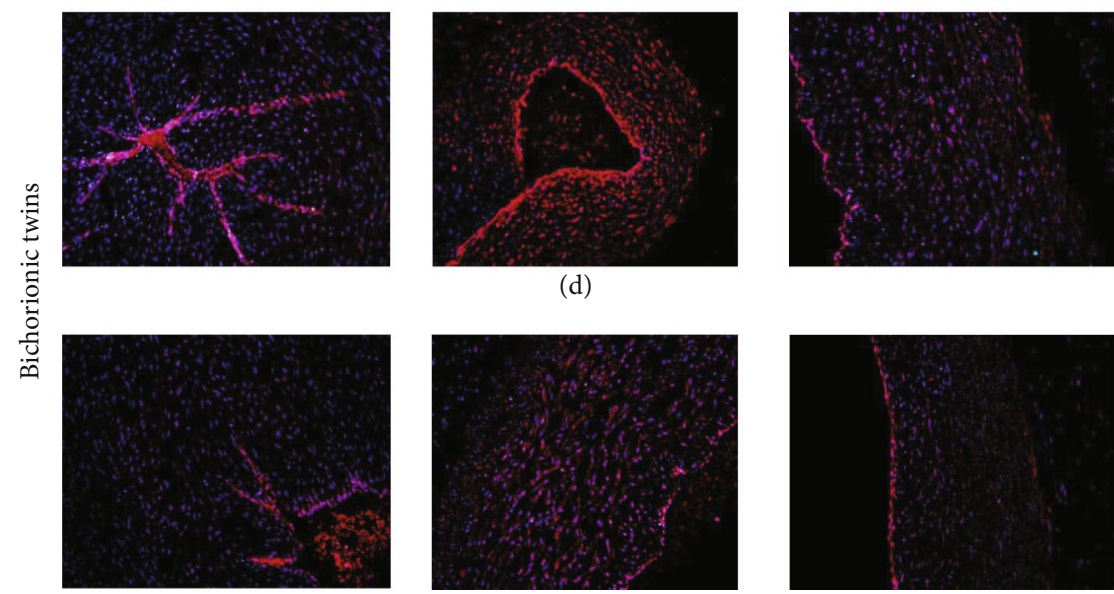

(d)
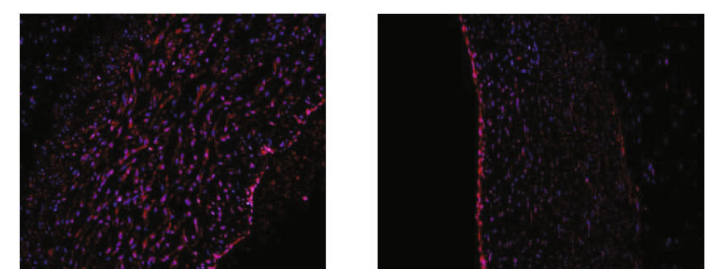

(e)

FIgURE 5: CALCA expression in the umbilical cord. Tissue section of umbilical cord were exposed to $\alpha \mathrm{CGRP}$ IgG at $4^{\circ} \mathrm{C}$ for $20 \mathrm{minutes}$. The localization of $\alpha$ CGRP was monitored by immunofluorescence microscopy. In umbilical cord tissue, CGRP was weakly expressed, mainly in vascular endothelial cell layer. (a), (b), and (c) are identical triplets ((c) is the invalid death case); (d) and (e) are fraternal twins.

percentage of methylation of $\mathrm{CpG}$ island in CALCB promoter region in $\mathrm{D}$ and $\mathrm{E}$ umbilical cord tissues of full-term fetuses was only $2.778 \%$ and $7.0 \%$. In addition, the methylation percentage of CALCB in umbilical cord tissues of premature fetuses was higher than that of term births, and the CALCB promoter region methylation percentage in the $C$ umbilical cord tissues of fetuses that died after rescue remained the highest (Figure 4).

Among these, the identical triplets were triplet $\mathrm{A}$, triplet $\mathrm{B}$, and triplet $\mathrm{C}$ and triplet $\mathrm{D}$ and triplet $\mathrm{E}$ fraternal twins. The results of pyrophosphorylation sequencing showed that the methylation levels of CALCA and CALCB in the umbilical cord tissue of three premature births with single chorionic sac were higher than those of full-term twins with double chorionic sac, and the methylation levels of death cases after rescue were higher when compared to others.

3.5. Tissue Immunohistochemical Test Results. Immunofluorescence results revealed that the expression of $\alpha$ CGRP in the umbilical cord of the twin brothers (triplet A, triplet B, and triplet C) was lower than that of normal controls (triplet $\mathrm{D}$ and triplet E), especially in death cases (triplet C) (Figure 5). Immunofluorescence results showed that the level of $\beta$ CGRP revealed no significant differences in the vascular endothelium and vascular wall of umbilical cord of 

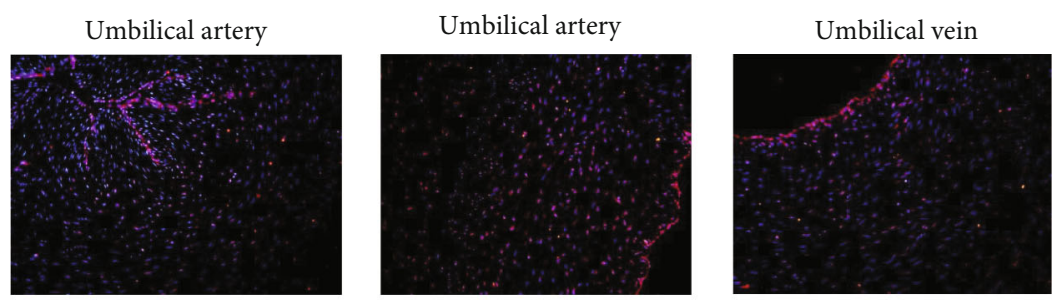

(a)
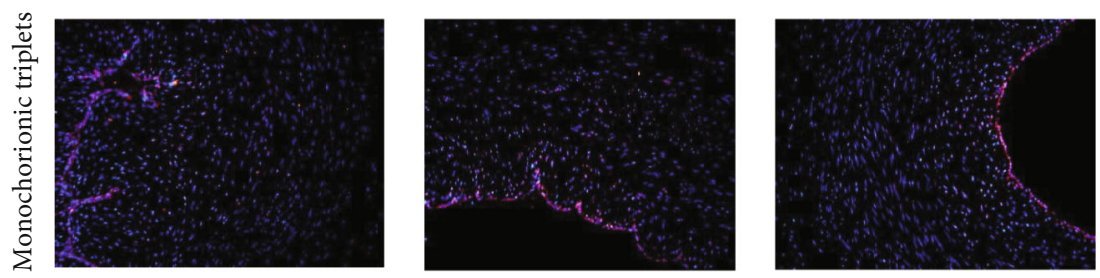

(b)
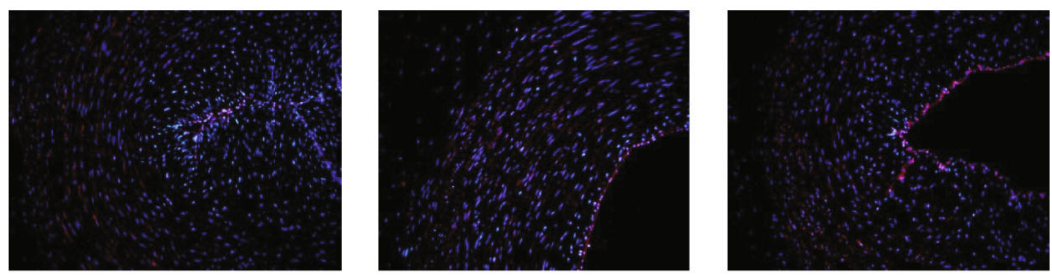

(c)
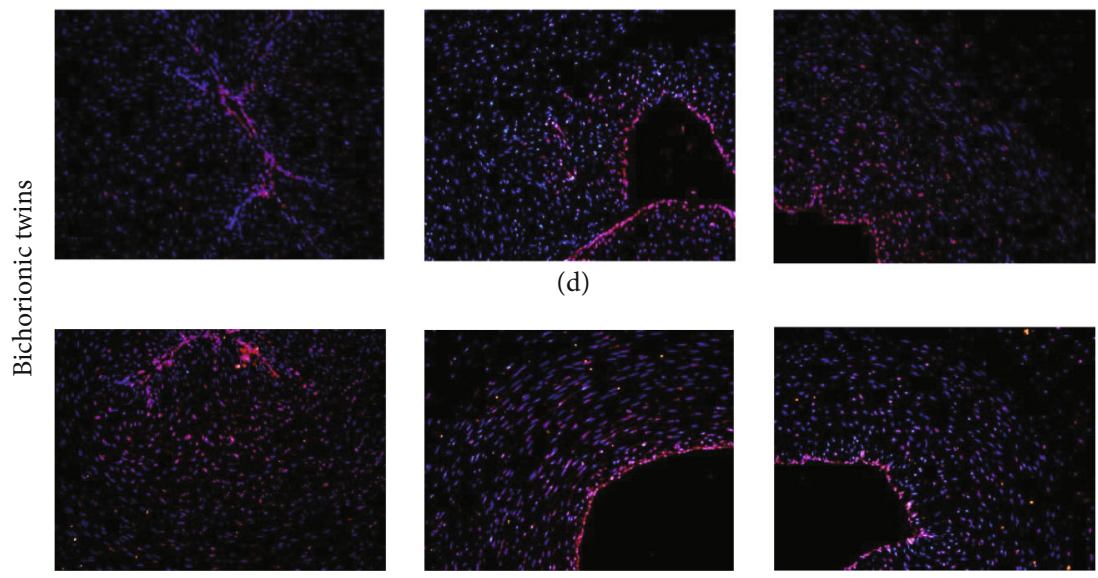

(d)
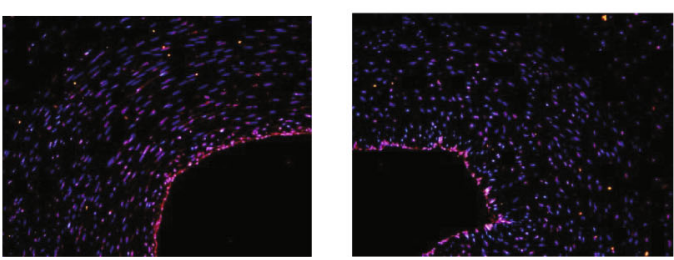

(e)

Figure 6: CALCB expression in the umbilical cord. Tissue section of umbilical cord were exposed to $\beta$ CGRP IgG at $4{ }^{\circ} \mathrm{C}$ for 20 minutes. The localization of $\beta$ CGRP was monitored by immunofluorescence microscopy. In umbilical cord tissue, CGRP was weakly expressed, mainly in vascular endothelial cell layer. (a), (b), and (c) are identical triplets; (d) and (e) are fraternal twins.

twin brothers and normal control, including the death cases without rescue (Figure 6).

\section{Discussion}

Premature infants are susceptible to many complications due to impaired immunity, inflammation, and vascular regulation $[13,14]$. The discovery with regard to the relationship between calcitonin/calcitonin gene-related peptide (CT/CGRP) and pregnancy highlighted new signal transmission mediators in various physiological processes including reproduction $[15,16]$. In addition to vascular regulation, CGRP comprises of a variety of functions in regulating immune and inflammatory responses [17-19]. It integrates inflammatory and immune responses in the local microenvironment $[5,6,15]$. CGRP can change lymphocyte proliferation, antigen presentation, cytokine production, B lymphocyte differentiation, and adhesion molecule expression $[5,6]$. Studying the effects of neuropeptides on inflammation and vascular regulation can help us to uncover the possible mechanisms in preterm infants.

Our previous study has shown that mutations or abnormal splicing of CGRP gene could lead to abnormal localization and synthesis of CGRP in cells, causing perineuritis and vasculitis [5]. Similar to vascular endothelial cells and smooth muscle cells, trophoblast cells also expressed 
receptor proteins such as CRLR and RAMP1, suggesting that CGRP might be involved in the regulation of trophoblast function [20]. Long-term administration of CGRP receptor antagonist $\mathrm{CGRP}_{8-37}$ into pregnant rats significantly reduced the weight of young rats, increased systolic blood pressure and fetal mortality in a dose-dependent manner [21, 22]. This phenomenon suggests the relationship between the expression of CGRP and premature birth.

Preterm birth is associated with DNA methylation in several different tissues, including placenta, neonatal blood, and neonate-birth saliva $[23,24]$. In these previous studies, many CpG loci associated with preterm birth were located in genes associated with neuronal development and/or neurodegenerative diseases $[25,26]$. This might be one of the reasons for the differences in the development of identical fetuses [27, 28]. In this study, pyrosequencing proved that CALCA and CALCB methylation levels of preterm umbilical cord tissues of single-chorionic triple-amniotic sac were higher than those of double-chorionic twins and normal controls as derived from the database. Moreover, the methylation levels of death cases after rescue were higher when compared to the other two twin brothers. Immunofluorescence results showed that the expression of $\alpha$ CGRP was decreased in the blood vessel wall of the umbilical cord of monozygotic triplets, especially in death cases, but no significant difference was observed in $\beta$ CGRP, whether it is compensatory or not requires further in-depth study.

CGRP hypermethylation might affect vascular adaptation of umbilical cord and function of trophoblast cells during pregnancy [29]. Therefore, we hypothesized whether high methylation of $\mathrm{CpG}$ island in the promoter region of CGRP gene in the umbilical cord tissues led to low expression of CGRP, promoting the development of preterm birth. The two subtypes of $\alpha$ CGRP and $\beta$ CGRP have extremely high similarities and differ only in three amino acids, where one of them is lacking, and the other changes positively or negatively [30, 31]. Compared with the wild-type control, the $\beta$ CGRP mRNA levels in the dorsal root ganglia and spinal cord of $\alpha$ CGRP knockout mice showed no change, but the levels in the intestine were reduced by two times [32]. In the intestines of mice lacking $\beta$ CGRP, the expression of $\alpha$ CGRP messenger RNA was increased, indicating an adaptive mechanism to compensate the lack of $\beta$ CGRP.

In conclusion, this study focused on premature live births of triple-chorionic tricotyledon after single-embryo transfer. The pyrosequencing method was used to detect the methylation of CALCA and CALCB in the umbilical cord and analyzed the normal control results of database search. This revealed that CALCA and CALCB hypermethylation might affect pregnancy vascular adaptation and trophoblast cell function by regulating the expression of CGRP. It is considered as an extremely important cause of premature birth and vasculitis.

\section{Data Availability}

All data and materials generated during and/or analysed during the current study are available from the corresponding author on reasonable request.

\section{Ethical Approval}

All experimental procedures in animal work were approved by the Ethical Committee of Fujian Medical University. This study was carried out in accordance with the recommendations of Medical ethics committee of the First Affiliated Hospital of Fujian Medical University. The protocol was approved by the Medical Ethics Committee of the First Affiliated Hospital of Fujian Medical University [036] and 2018[049].

\section{Disclosure}

The funding sources played key supportive role for sample collection, molecular analysis of patient samples, and bioinformatics analysis.

\section{Conflicts of Interest}

The authors have no competing interests to declare.

\section{Authors' Contributions}

Q.-C.L., F.G., and Y.-J.G. planned the project. Q.-C. L. and F.G. conceived and designed the study. X.-T.C., K.Z., Q.-Y.G., F.G., and Q.-C.L. performed the sample collection. S.-R. H., Q.-Q.C., H.-L.Z., Y.-L.Z., and Q.-C.L. performed immunohistochemistry. Q.-C.L., F.G., and H.-L.Z. performed the expression analysis. Q.-C.L., X.-M.X., and Q.-Y.G. analyzed the data and drafted the manuscript. All authors reviewed the manuscript and approved the final version. Feng Gao, Yujia Guo, Xingting Chen, and Qiuyang Gu contributed equally to this work.

\section{Acknowledgments}

We would like to thank PhD. Li Youzhu, Center of Reproductive Medicine, the First Affiliated Hospital of Xiamen University, for his support in embryological interpretation; $\mathrm{PhD}$. Xinqin He, Department of Obstetrics and Gynecology, 1st Affiliated Hospital, Fujian Medical University, for his support in tissue sample location; and the students (Yuxin Liu, Yue Luo, Xiaoxia Luo, and Junjie Lai) from the Department of Medical Laboratory of Fujian Medical University for their support in language processing. The authors sincerely thank the participants for their help and willingness to take part in this study. This work was supported by the National Natural Science Foundation of China (Nos. 82172953 and 81871293), Medical Innovation in Fujian Province (2019CX-27 and 2020CXA030), Fujian Medical University Sailing Fund Project (2018QH1078), Key Projects of Natural Science Foundation of Fujian (2021J02036), and Youth Fund of Fujian Provincial Department of Health (2019-1-41 and 2019-1-46).

\section{References}

[1] H. Rabe, G. M. L. Gyte, J. L. Díaz-Rossello, and L. Duley, "Effect of timing of umbilical cord clamping and other strategies to influence placental transfusion at preterm birth on 
maternal and infant outcomes," Cochrane Database of Systematic Reviews, vol. 9, article CD003248, 2019.

[2] I. Sohn, M. Sheykhzade, L. Edvinsson, and A. Sams, "The effects of CGRP in vascular tissue - classical vasodilation, shadowed effects and systemic dilemmas," European Journal of Pharmacology, vol. 881, p. 173205, 2020.

[3] C. Yallampalli, M. Chauhan, C. S. Thota, S. Kondapaka, and S. J. Wimalawansa, "Calcitonin gene-related peptide in pregnancy and its emerging receptor heterogeneity," Trends in Endocrinology and Metabolism, vol. 13, no. 6, pp. 263-269, 2002.

[4] Y. Dong, A. Betancourt, M. Chauhan et al., "Pregnancy increases relaxation in human omental arteries to the CGRP family of peptides," Biology of Reproduction, vol. 93, no. 6, p. 134, 2015.

[5] Q.-c. Liu, F. Chen, C.-y. Wu et al., "CALCB splice region pathogenic variants leading to plasma cell neurotropic enrichment in type 1 autoimmune pancreatitis," Cell Death \& Disease, vol. 8, no. 2, article e2591, 2017.

[6] Q. Liu, Y. Lin, S. Zhang et al., "CGRP-mediated prolactin upregulation: a possible pathomechanism in IgG4-related disease," Inflammation, vol. 44, no. 2, pp. 536-548, 2021.

[7] C. C. Chen, L. C. Chang, C. H. Yao et al., "Increased calcitonin gene-related peptide and macrophages are involved in Astragalus membranaceus-mediated peripheral nerve regeneration in rats," The American Journal of Chinese Medicine, vol. 46, no. 1, pp. 69-86, 2018.

[8] L. H. Peng, X. Q. Qin, R. R. Tan, C. Liu, H. J. Liu, and X. Qu, "Calcitonin gene-related peptide regulates the potential antigen uptake ability of human bronchial epithelial cells," Journal of Interferon \& Cytokine Research, vol. 38, no. 10, pp. 463-468, 2018.

[9] A. Lapillonne, E. Basson, G. Monneret, J. Bienvenu, and B. L. Salle, "Lack of specificity of procalcitonin for sepsis diagnosis in premature infants," Lancet, vol. 351, no. 9110, pp. 12111212, 1998.

[10] P. M. Broad, A. J. Symes, R. V. Thakker, and R. K. Craig, "Structure and methylation of the human calcitonin/alphaCGRP gene," Nucleic Acids Research, vol. 17, no. 17, pp. 6999-7011, 1989.

[11] N. Y. Souren, L. A. Gerdes, P. Lutsik et al., "DNA methylation signatures of monozygotic twins clinically discordant for multiple sclerosis," Nature Communications, vol. 10, no. 1, p. 2094, 2019.

[12] K. Im, S. Mareninov, M. F. P. Diaz, and W. H. Yong, "An introduction to performing immunofluorescence staining," Methods in Molecular Biology., vol. 1897, 2019.

[13] Y. Wang, L. Zhang, Y. Wu et al., "Peptidome analysis of umbilical cord mesenchymal stem cell (hUC-MSC) conditioned medium from preterm and term infants," Stem Cell Research \& Therapy., vol. 11, no. 1, p. 414, 2020.

[14] A. Katheria, F. Reister, J. Essers et al., "Association of umbilical cord milking vs delayed umbilical cord clamping with death or severe intraventricular hemorrhage among preterm infants," Journal of the American Medical Association, vol. 322, no. 19, pp. 1877-1886, 2019.

[15] S. K. Parida, D. B. Schneider, T. D. Stoss, T. H. Pauly, and J. P. McGillis, "Elevated circulating calcitonin gene-related peptide in umbilical cord and infant blood associated with maternal and neonatal sepsis and shock," Pediatric Research, vol. 43, no. 2, pp. 276-282, 1998.
[16] M. Chauhan, A. Betancourt, M. Balakrishnan et al., "Impaired vasodilatory responses of omental arteries to CGRP family peptides in pregnancies complicated by fetal growth restriction," The Journal of Clinical Endocrinology and Metabolism, vol. 101, no. 8, pp. 2984-2993, 2016.

[17] C. Schleithoff, S. Voelter-Mahlknecht, I. N. Dahmke, and U. Mahlknecht, "On the epigenetics of vascular regulation and disease," Clinical Epigenetics, vol. 4, no. 1, p. 7, 2012.

[18] B. Holzmann, "Antiinflammatory activities of CGRP modulating innate immune responses in health and disease," Current Protein \& Peptide Science, vol. 14, no. 4, pp. 268-274, 2013.

[19] M. D. Harzenetter, A. R. Novotny, P. Gais, C. A. Molina, F. Altmayr, and B. Holzmann, "Negative regulation of TLR responses by the neuropeptide CGRP is mediated by the transcriptional repressor ICER," Journal of Immunology, vol. 179, no. 1, pp. 607-615, 2007.

[20] Y. L. Dong, S. Vegiraju, M. Chauhan, and C. Yallampalli, "Expression of calcitonin gene-related peptide receptor components, calcitonin receptor-like receptor and receptor activity modifying protein 1 , in the rat placenta during pregnancy and their cellular localization," Molecular Human Reproduction, vol. 9, no. 8, pp. 481-490, 2003.

[21] Y. L. Dong, S. Vegiraju, M. Chauhan et al., "Involvement of calcitonin gene-related peptide in control of human fetoplacental vascular tone," American Journal of Physiology. Heart and Circulatory Physiology, vol. 286, no. 1, pp. H230-H239, 2004.

[22] P. R. R. Gangula, Y. L. Dong, S. J. Wimalawansa, and C. Yallampalli, "Infusion of pregnant rats with calcitonin gene-related peptide (CGRP)8-37, a CGRP receptor antagonist, increases blood pressure and fetal mortality and decreases fetal growth," Biology of Reproduction, vol. 67, no. 2, pp. 624629, 2002.

[23] X. M. Wang, F. Y. Tian, L. J. Fan, C. B. Xie, Z. Z. Niu, and W. Q. Chen, "Comparison of DNA methylation profiles associated with spontaneous preterm birth in placenta and cord blood," BMC Medical Genomics, vol. 12, no. 1, p. 1, 2019.

[24] T. P. York, S. J. Latendresse, C. Jackson-Cook et al., "Replicated umbilical cord blood DNA methylation loci associated with gestational age at birth," Epigenetics, vol. 15, no. 11, pp. 1243-1258, 2020.

[25] X. G. Wang and R. K. Bhandari, "DNA methylation dynamics during epigenetic reprogramming of medaka embryo," Epigenetics, vol. 14, no. 6, pp. 611-622, 2019.

[26] T. M. Everson, T. M. O’Shea, A. Burt et al., "Serious neonatal morbidities are associated with differences in DNA methylation among very preterm infants," Clinical Epigenetics, vol. 12, no. 1, p. 151, 2020.

[27] S. Mani and M. Mainigi, "Embryo culture conditions and the epigenome," Seminars in Reproductive Medicine, vol. 36, no. 3/4, pp. 211-220, 2018.

[28] B. Novakovic, S. Lewis, J. Halliday et al., "Assisted reproductive technologies are associated with limited epigenetic variation at birth that largely resolves by adulthood," Nature Communications, vol. 10, no. 1, p. 3922, 2019.

[29] Y. L. Dong, S. Vegiraju, and C. Yallampalli, "Ca2+ signaling in human fetoplacental vasculature: effect of CGRP on umbilical vein smooth muscle cytosolic Ca2+ concentration," American Journal of Physiology. Heart and Circulatory Physiology, vol. 289, no. 2, pp. H960-H967, 2005. 
[30] P. L. Tsiolaki, G. I. Nasi, F. A. Baltoumas et al., " $\alpha$ CGRP, another amyloidogenic member of the CGRP family," Journal of Structural Biology, vol. 203, no. 1, pp. 27-36, 2018.

[31] F. Gao, G. Liu, J. Wang et al., "Methylation of CALCA and CALCB in pancreatic ductal adenocarcinoma," Oxidative Medicine and Cellular Longevity., vol. 2021, article 2088345, pp. 1-13, 2021.

[32] B. Schütz, D. Mauer, A.-M. Salmon, J.-P. Changeux, and A. Zimmer, "Analysis of the cellular expression pattern of $\beta$ CGRP in $\alpha$-CGRP-deficient mice," The Journal of Comparative Neurology, vol. 476, no. 1, pp. 32-43, 2004. 